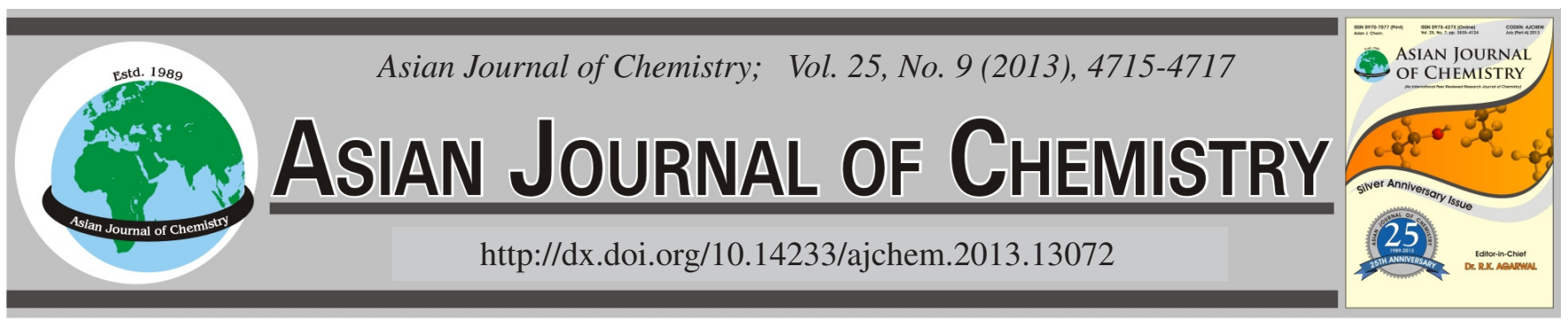

\title{
Analysis of Lattice Temperature Effects on a GaInP/6H-SiC Strained Quantum-Well Lasers
}

\section{SAeid MARJAni $^{1,{ }^{*},}$ Rahim FAeZ ${ }^{2}$ and Seyed Ebrahim Hosseini ${ }^{3}$}

${ }^{1}$ Young Researchers Club, Arak Branch, Islamic Azad University, Arak, Iran

${ }^{2}$ Department of Electrical Engineering, Sharif University of Technology, Tehran, Iran

${ }^{3}$ Department of Electrical Engineering, Ferdowsi University of Mashhad, Mashhad, Iran

*Corresponding author: E-mail: saeidmarjani@yahoo.com

(Received: 9 May 2012;

Accepted: 1 March 2013)

AJC-13055

In this paper, simulative study on the effects of lattice temperature on a GaInP/6H-SiC strained quantum-well (QW) laser's device is presented. Loss mechanisms are severe in the edge-emitting lasers. As a consequence, the wall-plug efficiency is sensitive to changes in temperature. The lasers used in this work were separate-confinement quantum-well lasers with a single strained GaInP, located in a latticematched waveguide core and cladding region of $6 \mathrm{H}-\mathrm{SiC}$. This paper provides the key results of the wall-plug efficiency upon the lattice temperature.

Key Words: Wall-plug efficiency, Lattice temperature, Silicon carbide polymers, Quantum-well lasers.

\section{INTRODUCTION}

Silicon carbide $(\mathrm{SiC})$ is a binary compound of IV-IV family. In structure of this material, each atom is surrounded by four different atoms, which are combined through targeted strong tetrahedral $s p^{3}$ key. As a result of its outstanding chemical and thermal stability, superior thermal conductivity and high breakdown field, $\mathrm{SiC}$ has been considered as a promising semiconductor material for high power, high-frequency and high temperature electronics and optoelectronic devices, where the existing Si or GaAs technology cannot provide any satisfactory performance ${ }^{1}$. This intensive research effort resulted in the commercialization of the first SiC-based devices in year 2001 namely, high power Schottky diodes ${ }^{2}$. Properties such as the large breakdown electric field strength, large saturated electron drift velocity, small dielectric constant, reasonably high electron mobility and high thermal conductivity make $\mathrm{SiC}$ an attractive candidate for fabricating power devices with reduced power losses and die size ${ }^{3}$.

Silicon carbide has many stable polytypes, including cubic zinc-blende, hexagonal and rhombohedral polytypes. In the cubic zinc-blende structure, labeled as $3 \mathrm{C}-\mathrm{SiC}$ or $\beta-\mathrm{SiC}, \mathrm{Si}$ and $\mathrm{C}$ occupy ordered sites in a diamond framework. In hexagonal polytypes $\mathrm{nH}-\mathrm{SiC}$ and rhombohedral polytypes $\mathrm{n}$ $\mathrm{R}-\mathrm{SiC}$, generally referred to as $\alpha \eta-\mathrm{SiC}, \mathrm{n}$ Si-C bilayers consisting of $\mathrm{C}$ and $\mathrm{Si}$ layers stack in the primitive unit cell. The lattice structures of the $3 \mathrm{C}-\mathrm{SiC}$ and $6 \mathrm{H}-\mathrm{SiC}$ phases, which are most important for this paper, are presented in Fig. $1^{4}$. (a)

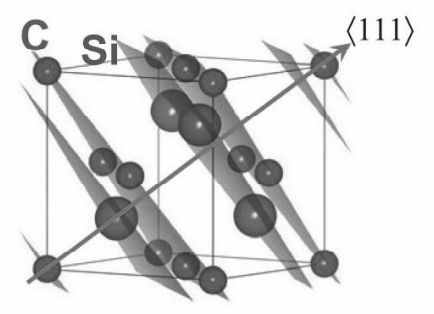

(b)

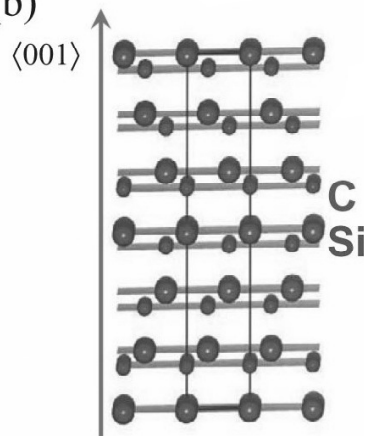

Fig. 1. (a) Unit cell of cubic 3C-SiC. (b) Four unit cells of hexagonal $6 \mathrm{H}-\mathrm{SiC}$

The use of strained quantum wells to improve the performance of semiconductor lasers was suggested by Adams ${ }^{5}$ and Yablonovich and Kane ${ }^{6}$ and strained wells are now usually used in such different material systems as InGaAs-GaAs, GaInP-AlGaInP and InGaN-GaN.

In this paper, we attempt to evaluate the effect of lattice temperature on the wall-plug efficiency of a GaInP/6H-SiC strained quantum-well(lasers using numerically-based simulation software ${ }^{7}$. In the following, we first briefly describe the numerical model and the details of the laser structure. Next we present the obtained numerical results. Finally, the conclusions provide common guidelines for designing performance of laser. 


\section{THEORY AND MODEL}

In modeling edge emitting laser, we must consider the electrical, optical and thermal interaction during laser performance. Thus base of simulation is to solve Poisson and continuity equations for electrons and holes ${ }^{7}$. Poisson's equation is defined by eqn. 1 :

$$
\nabla \cdot(\varepsilon \nabla \Psi)=\rho
$$

where $\Psi$ is electrostatic potential, $\rho$ is local charge density and $\varepsilon$ is local permittivity. The continuity equations of electron and hole are given by ${ }^{8}$ eqn. 2 and 3:

$$
\begin{aligned}
& \frac{\mathrm{dn}}{\mathrm{dt}}=\mathrm{G}_{\mathrm{n}}-\mathrm{R}_{\mathrm{n}}+\frac{1}{\mathrm{q}} \nabla \cdot \mathrm{j}_{\mathrm{n}} \\
& \frac{\mathrm{dp}}{\mathrm{dt}}=\mathrm{G}_{\mathrm{p}}-\mathrm{R}_{\mathrm{p}}+\frac{1}{\mathrm{q}} \nabla \cdot \mathrm{j}_{\mathrm{p}}
\end{aligned}
$$

where $\mathrm{n}$ and $\mathrm{p}$ are the electron and hole concentration, $\mathrm{J}_{\mathrm{n}}$ and $J_{p}$ are the electron and hole current densities, $G_{n}$ and $G_{p}$ are the generation rates for electrons and holes, $R_{n}$ and $R_{p}$ are the recombination rates and $\mathrm{q}$ is the magnitude of electron charge.

The fundamental semiconductor equations 1-3 are solved self-consistently together with Helmholtz and the photon rate equations. The applied technique for solution of Helmholtz equation is based on improved effective index $\operatorname{model}^{9}$ which shows accuracy for great portion of preliminary problems. This model is very good adapted to simulation of laser structures and it is often called effective frequency method ${ }^{10}$.

Two-dimensional Helmholtz equation is solved to determine the transverse optical field profile and it is given by ${ }^{7}$ :

$$
\nabla^{2} \mathrm{E}(\mathrm{r}, \mathrm{z}, \varphi)+\frac{\omega_{0}}{\mathrm{c}^{2}} \varepsilon(\mathrm{r}, \mathrm{z}, \varphi, \omega) \mathrm{E}(\mathrm{r}, \mathrm{z}, \varphi)=0
$$

where $\omega$ is the frequency, $\varepsilon(\mathrm{r}, \mathrm{z}, \varphi, \omega)$ is the complex dielectric permittivity, $\mathrm{E}(\mathrm{r}, \mathrm{z}, \varphi)$ is the optical electric field and $\mathrm{c}$ is the speed of light in vacuum. The light power equation relates electrical and optical models. The photon rate equation is given by $^{7}$ eqn. 5 :

$$
\frac{\mathrm{dS}}{\mathrm{dt}}=\left(\frac{\mathrm{c}}{\mathrm{N}_{\mathrm{eff}}} \mathrm{G}_{\mathrm{m}}-\frac{1}{\tau_{\mathrm{ph}_{\mathrm{m}}}}-\frac{\mathrm{cL}}{\mathrm{N}_{\mathrm{eff}}}\right) \mathrm{S}_{\mathrm{m}}+\mathrm{R}_{\mathrm{sp}_{\mathrm{m}}}
$$

where $S_{m}$ is the photon number, $G_{m}$ is the modal gain, $R_{\text {spm }_{m}}$ is the modal spontaneous emission rate, $\mathrm{L}$ represents the losses in the laser, $\mathrm{N}_{\text {eff }}$ is the group effective refractive index, $\tau_{\mathrm{ph}_{\mathrm{m}}}$ is the modal photon lifetime and $\mathrm{c}$ is the speed of light in vacuum.

The heat flow equation has the form ${ }^{7}$ :

$$
\mathrm{C} \frac{\partial \mathrm{T}_{\mathrm{L}}}{\partial \mathrm{t}}=\nabla\left(\kappa \nabla \mathrm{T}_{\mathrm{L}}\right)+\mathrm{H}
$$

where $\mathrm{C}$ is the heat capacitance per unit volume, $\kappa$ is the thermal conductivity, $\mathrm{H}$ is the generation, $\mathrm{T}_{\mathrm{L}}$ is the local lattice temperature and $\mathrm{H}$ is the heat generation term.

Eqns. 1-6 provide an approach that can account for the mutual dependence of electrical, thermal, optical and elements of heat sources. In this paper, we employ numerical-based simulation software to assist in the device design and optimization ${ }^{7}$.

Fig. 2 shows the schematic design of $0.83 \mu \mathrm{m}$ strained quantum-well laser diode device. The lasers used in this work

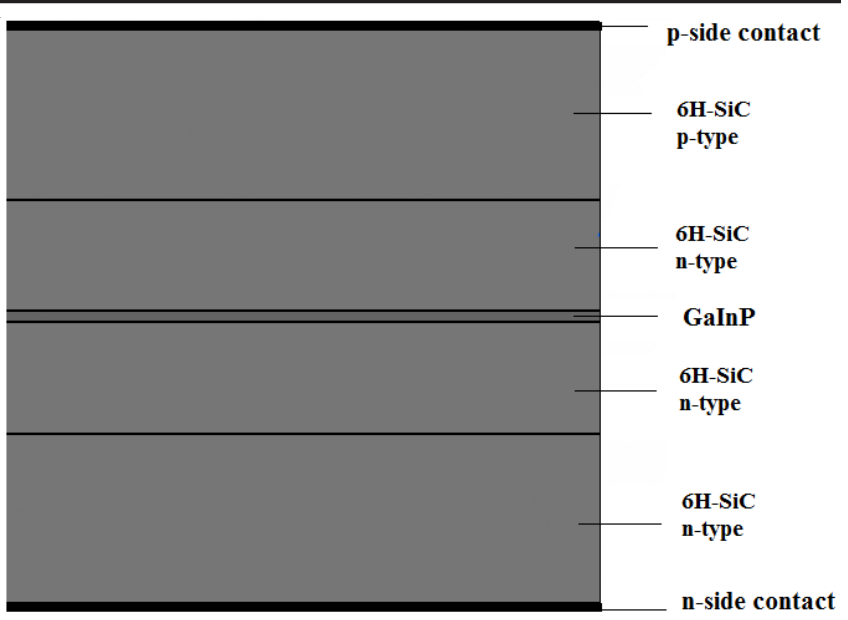

Fig. 2. Schematic structure of the laser device

were separate-confinement quantum-well lasers with a single strained GaInP quantum well, $10 \mathrm{~nm}$ wide, located in a latticematched waveguide core and cladding region of $6 \mathrm{H}-\mathrm{SiC}$ with wide of layers $95 \mathrm{~nm}$ and $1500 \mathrm{~nm}$ respectively.

\section{RESULTS AND DISCUSSION}

Heat loss from the modeled photon crystal verticalcavity surface-emitting diode lasers (PhC VCSEL) device was specified using thermal contacts at the top electrode, bottom electrode and the device sidewall. The thermal contacts define thermal conductivities to simulate heat loss from radiation via exposed surfaces or conduction through the semiconducting material to a heatsink.

The wall-plug efficiency is highest importance for many applications. The wall-plug efficiency equation is given by ${ }^{11}$ eqn. 7:

$$
\eta_{\mathrm{wp}}=\frac{\mathrm{P}_{\text {out }}}{\mathrm{P}_{\text {in }}}=\frac{\mathrm{P}_{\text {out }}}{\mathrm{IV}}
$$

where $\mathrm{P}_{\text {out }}$ is the output optical power, I is the injection current and $\mathrm{V}$ is bias voltage.

The lattice temperature was varied between $280 \mathrm{~K}$ until $350 \mathrm{~K}$ and its effect on the wall-plug efficiency curve is shown in Fig. 3. As can be seen from Fig.3, increasing of the lattice temperature causes the reduction of the wall-plug efficiency. This decrement should be mainly due to the combined effects of Auger processes, valence band absorptions and free carrier absorptions. At high temperature, electrons leak out of the quantum well into the waveguide core due to the small conduction band offset. Unbalanced charge distribution between the quantum well and the waveguide core causes an electric field that traps holes in the waveguide core and increases the internal absorption loss and recombination. Furthermore, Fig. 3 shows a decrement in the wall-plug efficiency $(4.1154 \%$ at $7 \mathrm{~V}$ to $1.3846 \%$ at $10 \mathrm{~V}$ at $280 \mathrm{~K}$ ) with increment in operating voltage.

\section{Conclusion}

This article described the lattice temperature have a profound impact on a $\mathrm{GaInP} / 6 \mathrm{H}-\mathrm{SiC}$ strained quantum-well (QW) lasers the wall-plug efficiency. The results indicate that increasing of the lattice temperature causes the reduction of the wall-plug efficiency. 


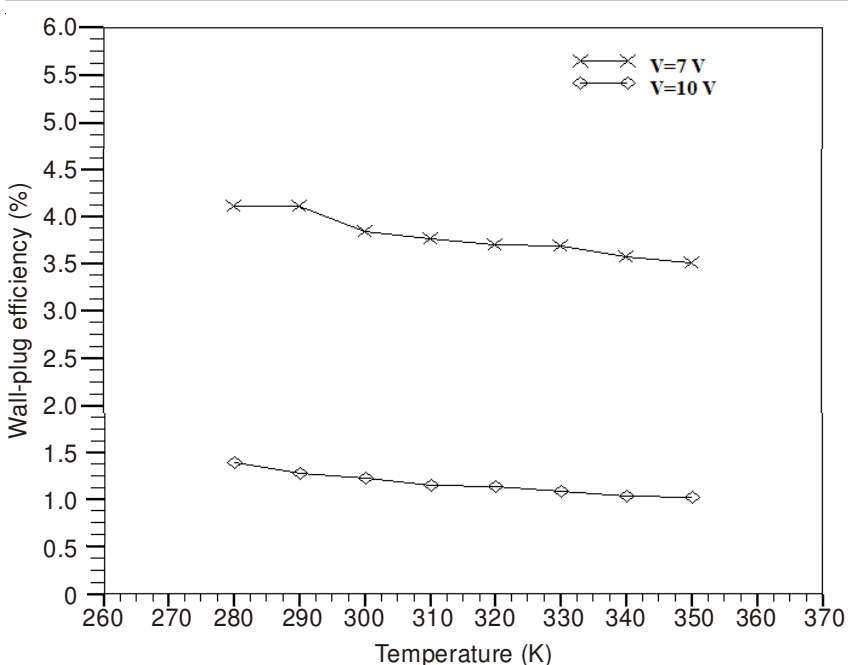

Fig. 3. Wall-plug efficiency versus lattice temperature as a function of bias voltage

\section{REFERENCES}

1. P.B. Klein, J. Appl. Phys., 103, 033702 (2008).

2. P.G. Neudeck, in eds.: K.H.J. Bushchow, R.W. Cahn, M.C. Flemings, B. Ilschner, E.J. Kramer and S. Mahajan, Encyclopedia of Materials: Science and Technology, Elsevier Science, Vol. 9, p. 8508 (2001).

3. M. Bhatnagar and B.J. Baliga, IEEE Transactions on Electron Devices, p. 40, (1993).

4. T. Muranaka, Y. Kikuchi, T. Yoshizawa, N. Shirakawa and J. Akimitsu, Sci. Technol. Adv. Mater., 9, 044204 (2008).

5. A.R. Adams, J. Electron. Lett., 22, 249 (1986).

6. E. Yablonovich and E.O. Kane, J. Lightwave Technol., LT-4, 504 (1986).

7. SILVACO International, ATLAS User's Manual, USA, SILVACO International Incorporated (2010).

8. J. Piprek, Semiconductor Optoelectronic Devices: Introduction to Physics and Simulation, Ch. 3 Carrier Transport and Ch. 6 Heat Generation and Dissipation UCSB: Academic Press, pp. 49-50 and 141-147 (2003).

9. G.R. Hadley, J. Opt. Lett., 20, 1483 (1995).

10. H. Wenzel and H.J. Wunsche, IEEE J. Quantum Electron., 33, 1156 (1997).

11. K. Iga, IEEE J Select. Topics Quantum Electron, 6, 1201 (2000).

12. Y. Suematsu and A.R. Adams, Handbook of Semiconductor Lasers and Photonic Integrated Circuits, Chapman \& Hall (1994). 